\title{
The Association of Co-Morbidities and Severity of Dengue Fever and Organ Specific Complications in Trivandrum District in Urban Kerala
}

\author{
Anila Richard ${ }^{1}$, Ratheesh Narayanan Santhanavally², Ravikumar Kurup ${ }^{3}$ \\ 1,2,3 Department of General Medicine, Government Medical College, Thiruvananthapuram, Kerala, India.
}

\section{ABSTRACT}

\section{BACKGROUND}

Dengue fever (DF) is caused by a flavivirus and is transmitted to humans by the vector Aedes aegypti. Industrialization and unplanned urbanization have led to an increase in incidence of DF. DF can lead to organ-specific complications especially in those with co-morbidities. The present study was done to estimate the prevalence of organ-specific complications in DF and determine the association of comorbidities and development of organ-specific complications.

\section{METHODS}

This is a prospective cross-sectional observational study. 148 participants with DF as confirmed by NS1 antigen or dengue IgM presenting to medicine outpatient department of Government Medical College, Trivandrum were enrolled in the study after obtaining written informed consent and obtaining Institutional Ethics Committee approval. Examination findings, laboratory investigations [complete blood count (CBC), liver \& renal function tests (RFT)], chest radiograph, ultrasonography (USG), magnetic resonance imaging (MRI), and cerebrospinal fluid (CSF) examination were done as routine procedures wherever necessary, and the details were collected in case record forms. Data was analysed using Rafter assessment of normality and homogeneity and chi square test was used to determine the association between parameters and organ specific complications. $\mathrm{P}$ $<0.05$ was considered statistically significant.

\section{RESULTS}

Acalculous cholecystitis (29.1), hepatitis (4.7 \%), aseptic meningitis (4.1\%), encephalopathy (4.1\%), myocarditis (3.4\%), encephalitis (2.7\%), acute kidney injury (2\%), acute respiratory distress syndrome $(2 \%)$, pericardial effusion $(1.4$ $\%)$, pleural effusion (1.4\%) and conduction anomalies of heart $(0.7 \%)$ were the organ specific complications associated with DF. Participants with co-morbidities were at a higher risk of developing organ-specific complications when compared to the healthier individuals.

\section{CONCLUSIONS}

Acalculous cholecystitis was the most common complication associated with DF and the presence of co-morbidities was a significant risk for development of complications. Proper planning for vector control measures especially during highrisk seasons would reduce the transmission of the disease and reduce the healthcare burden, mortality and morbidity associated with dengue fever.

\section{KEY WORDS}

Dengue Fever, Organ Specific Complications, Acalculous Cholecystitis, Hepatitis, Meningitis
Corresponding Author: Dr. Ratheesh Narayanan Santhanavally, Karthika, CRA-79, Kazhakuttom. P.O., Thiruvananthapuram-695582,

Kerala, India.

E-mail: ratishkarthika@yahoo.co.in

\section{DOI: $10.14260 /$ jemds/2021/786}

How to Cite This Article:

Richard A, Santhanavally RN, Kurup R. The association of co-morbidities and severity of dengue fever and organ specific complications in Trivandrum district in Urban Kerala. J Evolution Med Dent Sci 2021;10(45):3889-3894, $10.14260 /$ jemds/2021/786

Submission 03-11-2021,

Peer Review 12-11-2021,

Acceptance 03-12-2021, Published 28-12-2021.

Copyright (C) 2021 Anila Richard et al. This is an open access article distributed under Creative Commons Attribution License [Attribution 4.0 International (CC BY 4.0)] 


\section{BACKGROUND}

Dengue virus belongs to genus flavivirus and the family flaviviridae. The disease is arthropod-borne (spread by Aedes aegypti) and is fast spreading and depending on the antigenic subtypes, can be classified as dengue virus 1 to 4 . Dengue fever is the most common arboviral infection globally. ${ }^{1} \mathrm{DF}$ can be asymptomatic and self-limiting in some patients. According to World Health Organization (WHO) guidelines of 1997, dengue virus infections were classified into three categories as DF, dengue haemorrhagic fever (DHF), and dengue shock syndrome (DSS). Later this classification was revised, and patients were categorized based on the severity levels of the infection into DF without warning signs, DF with warning signs, and severe DF. The warning signs of DF include lethargy, persistent vomiting, abdominal tenderness or pain, mucosal bleeding, hepatomegaly, and thrombocytopenia with elevated hematocrit.2,3 DF is characterized by high-grade fever for 3 to 7 days, severe headache, myalgia, retro-orbital pain, and joint pain ${ }^{4}$ and is associated with transient flushing erythema in the initial 2 days of fever which changes to a maculopapular/morbilliform rash after $3-7$ days of fever. ${ }^{5}$ DHF mostly develops as a secondary dengue infection in adults, however, in infants, it may occur as a primary infection. ${ }^{6}$ Symptoms include high-grade fever with ecchymosis, epistaxis, purpura, gingival and mucosal bleeding and platelet count of less than $1 \mathrm{lakh} / \mathrm{mm}^{3}$ is usually associated with it. DSS is characterized by DHF along with cold clammy skin, intraoral cyanosis, and narrow pulse pressure ( $<20 \mathrm{~mm}$ of $\mathrm{Hg}$ ). DSS can lead to complications such as disseminated intravascular coagulation (DIC), multi-organ dysfunction syndrome, and shock. ${ }^{7}$ Subsequent infection with a heterologous strain increases the risk of developing DHF. ${ }^{8}$ In tropical and subtropical countries, over 2.5 billion people are infected with DF which includes residents and travellers, and is considered an endemic more than 100 countries. $^{9}$ Though India is considered as a hot spot for DF, the number of cases and complications are largely underestimated. ${ }^{10}$ Hence, an active and efficient surveillance system is essential for the detection, diagnosis, and prevention of DF.

The multisystemic complications associated with DF include encephalitis, encephalopathy, cardiomyopathy, hepatic disorders, orchitis, oophoritis, and pneumonia. ${ }^{11}$ Published literature has also demonstrated the occurrence of opsoclonus -myoclonus syndrome and brachial neuritis with DF in the Indian population. ${ }^{12}$ The cardiac manifestations associated with dengue are asymptomatic sinus bradycardia, transient AV blocks, transient ventricular arrhythmias, myocarditis, and pericardial effusion. ${ }^{13}$ But in reality, most of the cardiac manifestations are poorly investigated and are underreported. Signs of liver dysfunction such as elevated liver enzymes can be observed in sever $\mathrm{DF}^{14}$ and the common hepatic complications are direct liver damage, 15 hypoproteinaemia, hypoalbuminemia and coagulation anomalies.16 Myalgia, myositis, rhabdomyolysis, and hypokalaemic paralysis are the manifestations of muscle involvement in DF. ${ }^{17}$ Renal complications such as acute renal failure, glomerulonephritis, haematuria, and proteinuria can be associated with severe DF. ${ }^{18}$ Pneumonitis, pleural effusion, pulmonary haemorrhages, and acute respiratory distress syndrome (ARDS) are the commonly reported respiratory complications of DF.19 Involvement of the eyes in form of retinal haemorrhages, macular oedema, foveolitis, vasculitis, and optic neuropathy can be seen associated with DF.20

The presence of co-morbidities such as cardiovascular diseases, endocrine diseases, haematological abnormalities, liver disorders, renal dysfunction, autoimmune disorders and solid organ transplantation increases the risk of severe infections. ${ }^{21} \mathrm{DF}$ in patients with DM and DM with coexisting hypertension increases the chance of developing DHF/DSS. ${ }^{22}$ The presence of co-morbidities increase the risk of severity in dengue patients and increases incidence of organ-specific complications. Owing to the scarcity of published literature, the present study determines the association of comorbidities and severity of DF and organ specific complications associated with it.

\section{METHODS}

The present prospective cross-sectional observational study enrolled 148 participants with DF admitted in General Medicine Department of Government Medical College, Thiruvananthapuram from 2019 to 2020. Study commenced after obtaining Institutional Ethics Committee and written informed consent was obtained from all study participants. Sample size was calculated assuming an $\alpha$ error of $0.05, \beta$ error of 0.2 and $80 \%$ power. All participants aged 18 years and above admitted in the ward or intensive care unit (ICU) with dengue NS1Ag/ dengue IgM positive results were included in our study. The research study variables collected include age, gender, history of co-morbidities (Diabetes, hypertension, dyslipidaemia, chronic liver disease, chronic kidney disease, reactive airway disease), laboratory investigations such as platelet count, serum bilirubin, liver enzymes (SGOT and SGPT), blood urea, serum creatinine, electrocardiogram (ECG), chest x-ray. Computed tomography (CT) scan was performed in participants with neurological symptoms or headache. Data was collected in semi structured proforma in which age, sex, history of co-morbidities, symptoms of the patient were recorded. In addition to this laboratory investigation reports, previous reports of participants were also collected for analysis. Normality of data was assessed using the Shapiro-Wilk test and values are expressed as proportions and data is expressed as tables and bar diagrams. Chi square test was used to determine the association between parameters and a $\mathrm{P}<0.05$ was considered statistically significant.

\section{RESULTS}

$64.2 \%(n=95)$ participants were males and $35.8 \%(n=53)$ were females. Baseline characteristics of the study participants are demonstrated in table 1 . Co-morbidities of the study participants is demonstrated in figure $1.45 .9 \%$ participants were in the age between 21 to 30 years. Prevalence was slightly higher in rural areas compared to urban areas. Abnormalities in ECG were observed in $4.7 \%$ study participants and the causes of these abnormalities were myocarditis and conduction anomalies. Echocardiogram was done in nine participants who had chest pain as a presenting 
symptom and $4.7 \%$ participants had detectable abnormalities in echocardiogram. Five participants had global left ventricular hypokinesia suggestive of myocarditis and two participants had pericardial effusion. Chest X-ray demonstrated pleural effusion in two participants and pericardial effusion in two participants and one participant had X-ray findings consistent with ARDS. $1.4 \%$ participants had regions of focal involvement of brain parenchyma suggestive of encephalitis in CT scan. $7.4 \%$ participants had CSF findings diagnostic of aseptic meningitis or encephalitis. Among the study participants', fever was the most common symptom (98.6\%) followed by headache (50\%), vomiting (45.4\%), abdominal pain (32.4\%). Other symptoms included altered sensorium (4.1\%), breathlessness ( $4.1 \%)$, chest pain (3.4\%), cough (3.4\%), seizures (2.7\%) and oliguria (2\%).

\begin{tabular}{|ccc|}
\hline Parameter & Classification & N (\%) \\
Gender & Male & $95(64.2)$ \\
& Female & $53(35.8)$ \\
Location & Rural & $79(53.4)$ \\
& Urban & $69(46.6)$ \\
ECG & Normal & $141(95.3)$ \\
& Abnormal & $7(4.7)$ \\
Echocardiogram & Normal & $2(1.4)$ \\
& Abnormal & $7(4.7)$ \\
Chest X ray & Not done & $139(93.9)$ \\
& Normal & $143(96.6)$ \\
CT Brain & Abnormal & $5(3.4)$ \\
& Normal & $43(29.1)$ \\
& Abnormal & $2(1.4)$ \\
Ultrasonography of & Not Done & $103(69.6)$ \\
abdomen & Acalculous cholecystitis & $31(20.9)$ \\
& Hepatomegaly & $8(5.4)$ \\
& Splenomegaly & $1(0.7)$ \\
& Acalculous cholecystitis with hepatomegaly & $12(8.1)$ \\
CSF study & Acalculous cholecystitis with & $3(2)$ \\
& hepatosplenomegaly & $9(6.1)$ \\
& Normal & $11(7.4)$ \\
Platelet count & Abnormal & $128(86.5)$ \\
& Not done & $58(39.2)$ \\
\hline Table 1. Baseline Parameters of the Study Participants \\
\hline
\end{tabular}

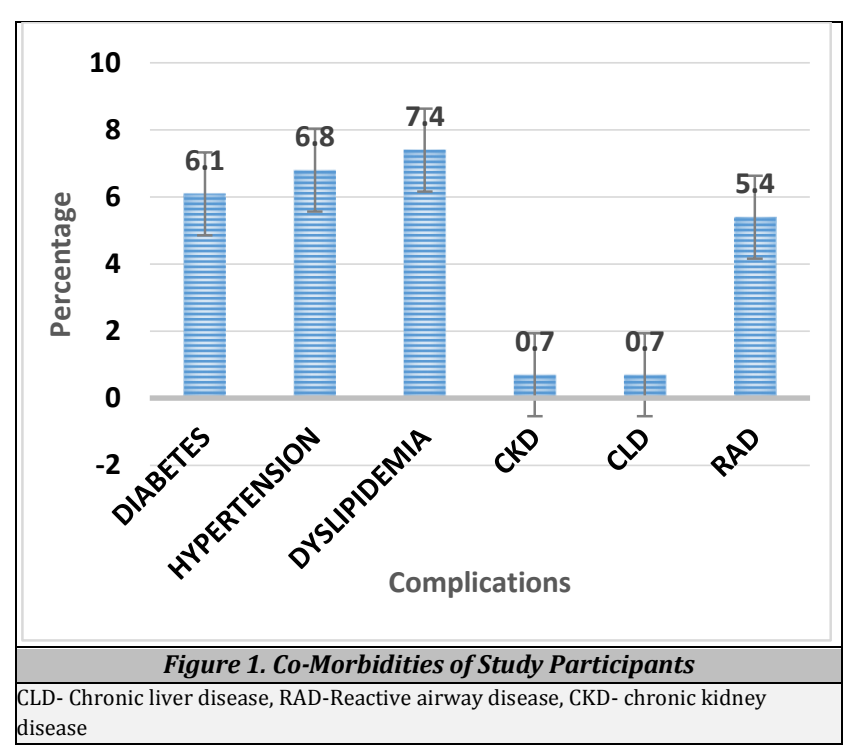

\begin{tabular}{|c|c|c|c|c|}
\hline \multirow{2}{*}{ Parameter } & \multirow{2}{*}{ Category } & \multicolumn{2}{|c|}{$\begin{array}{l}\text { Organ-Specific } \\
\text { Complication }\end{array}$} & \multirow[b]{2}{*}{$\mathbf{P}$} \\
\hline & & $\begin{array}{c}\text { No, } \\
\text { N (\%) }\end{array}$ & $\begin{array}{c}\text { Yes, } \\
\text { N (\%) }\end{array}$ & \\
\hline \multirow{5}{*}{ Age (Years) } & $<20$ & $11(55)$ & $9(45)$ & \multirow{5}{*}{0.1} \\
\hline & $21-30$ & $39(57.4)$ & 29 (42.6) & \\
\hline & $31-40$ & $13(43.3)$ & $17(56.7)$ & \\
\hline & $41-50$ & $13(72.2)$ & $5(27.8)$ & \\
\hline & $51-60$ & $10(83.3)$ & $2(16.7)$ & \\
\hline \multirow{4}{*}{$\begin{array}{c}\text { Platelet } \\
\text { count } \\
\text { (cells } / \mathrm{mm}^{3} \text { ) }\end{array}$} & $\leq 20000$ & $41(70.7)$ & $17(29.3)$ & \multirow{4}{*}{0.002} \\
\hline & $20001-50000$ & $41(56.9)$ & 31 (43.1) & \\
\hline & $50001-100000$ & $4(30.8)$ & $9(69.2)$ & \\
\hline & $>\quad 100000$ & 0 & $5(100)$ & \\
\hline \multirow[b]{2}{*}{ Gender } & Male & $53(55.8)$ & $42(44.2)$ & \multirow[b]{2}{*}{0.4} \\
\hline & Female & $33(62.3)$ & $20(37.7)$ & \\
\hline \multirow{2}{*}{ Location } & Rural & $41(51.9)$ & $38(48.1)$ & \multirow{2}{*}{0.1} \\
\hline & Urban & 45 (65.2) & $24(34.8)$ & \\
\hline \multirow{2}{*}{ ECG } & Normal & $86(61)$ & $55(39)$ & \multirow{2}{*}{0.001} \\
\hline & Abnormal & 0 & $7(100)$ & \\
\hline \multirow{3}{*}{$\begin{array}{l}\text { Echocardio } \\
\text { gram }\end{array}$} & Normal & $1(50)$ & $1(50)$ & \multirow{3}{*}{0.006} \\
\hline & Abnormal & 0 & $7(100)$ & \\
\hline & Not done & $85(61.2)$ & $54(38.8)$ & \\
\hline \multirow{2}{*}{ Chest X ray } & Normal & $86(60.1)$ & 57 (39.9) & \multirow{2}{*}{0.007} \\
\hline & Abnormal & 0 & $5(100)$ & \\
\hline \multirow{3}{*}{ CT Brain } & Normal & $13(30.2)$ & $30(69.8)$ & \multirow{3}{*}{$<0.001$} \\
\hline & Abnormal & 0 & $2(100)$ & \\
\hline & Not done & $73(70.9)$ & $30(29.1)$ & \\
\hline \multirow{7}{*}{$\begin{array}{c}\text { USG } \\
\text { abdomen }\end{array}$} & Normal & $56(81.2)$ & $13(18.8)$ & \\
\hline & Acalculous cholecystitis & $3(9.7)$ & $28(90.3)$ & \\
\hline & Hepatomegaly & $5(62.5)$ & $3(37.5)$ & \\
\hline & Splenomegaly & $1(100)$ & 0 & \\
\hline & $\begin{array}{c}\text { Acalculous cholecystitis } \\
\text { with Hepatomegaly }\end{array}$ & 0 & $12(100)$ & $<0.001$ \\
\hline & Acalculous cholecystitis & 0 & $3(100)$ & \\
\hline & $\begin{array}{l}\text { Witn nepatosplenomegary } \\
\text { Not done }\end{array}$ & $21(87.5)$ & $3(12.5)$ & \\
\hline & Normal & $2(22.2)$ & 7 (77.8) & \\
\hline CSF study & Abnormal & $1(9.1)$ & $10(90.9)$ & $<0.001$ \\
\hline & Not done & $83(64.8)$ & 45 (35.2) & \\
\hline Serum & $\leq 1.2$ & $84(64.1)$ & 47 (35.9) & \\
\hline bilirubin & $1.3-2.0$ & $2(20)$ & $8(80)$ & $<0.001$ \\
\hline$(\mathrm{mg} / \mathrm{dL})$ & $\geq 2$ & 0 & $7(100)$ & \\
\hline & $\leq 40$ & $54(79.4)$ & $14(20.6)$ & \\
\hline $\begin{array}{c}\text { SGOT } \\
\text { (Units/L) }\end{array}$ & $>\quad 40$ & $32(40)$ & $48(60)$ & $<0.001$ \\
\hline SGPT & $\leq 40$ & $54(79.4)$ & $14(20.6)$ & \\
\hline (Units/L) & $>\quad 40$ & $32(40)$ & $48(60)$ & $<0.001$ \\
\hline & $\leq 20$ & $15(78.9)$ & $26(21.1)$ & \\
\hline Blood urea & $21-40$ & $71(56.3)$ & $33(43.7)$ & 0.02 \\
\hline & $>\quad 40$ & 0 & $3(100)$ & \\
\hline Serum & $\leq 6$ & $52(66.7)$ & $26(33.3)$ & \\
\hline creatinine & $0.7-1.2$ & $34(50.7)$ & $33(49.3)$ & 0.02 \\
\hline$(\mathrm{mg} / \mathrm{dL})$ & $1.3+$ & 0 & $3(100)$ & \\
\hline Serum & $\leq 80$ & $86(60.6)$ & $56(39.4)$ & \\
\hline $\begin{array}{l}\text { amylase } \\
\text { (Units/L) }\end{array}$ & $81-100$ & 0 & $6(100)$ & 0.003 \\
\hline $\begin{array}{l}\text { Serum } \\
\text { lipase } \\
\text { (Units/L) }\end{array}$ & $\leq 160$ & $86(58.1)$ & $62(41.9)$ & $<0.001$ \\
\hline Table 2. & $\begin{array}{r}\text { of Organ-Sp } \\
\text { Para }\end{array}$ & $\begin{array}{l}\text { ic Comp } \\
\text { ers }\end{array}$ & ns wit & seline \\
\hline
\end{tabular}

\begin{tabular}{|cccc|}
\hline Co-Morbidities & \multicolumn{2}{c|}{ Organ-Specific Complication } & \multirow{2}{*}{ P } \\
& No, $\mathbf{n}(\%)$ & Yes, $\mathbf{n}(\%)$ & 0.009 \\
Diabetes & $9(100)$ & 0 & 0.1 \\
Hypertension & $8(80)$ & $2(20)$ & 0.09 \\
Dyslipidaemia & $9(81.8)$ & $2(18.2)$ & 0.4 \\
CKD & $1(100)$ & 0 & 0.4 \\
CLD & $1(100)$ & 0 & 0.08 \\
\hline RAD & $7(87.5)$ & 1 & \\
\hline Table 3. Comparison of Co-Morbidities and Organ-Specific \\
Complication \\
\hline
\end{tabular}

CKD- chronic kidney disease, CLD- chronic liver disease, RAD- reactive airway disease, association was determined using chi square test 


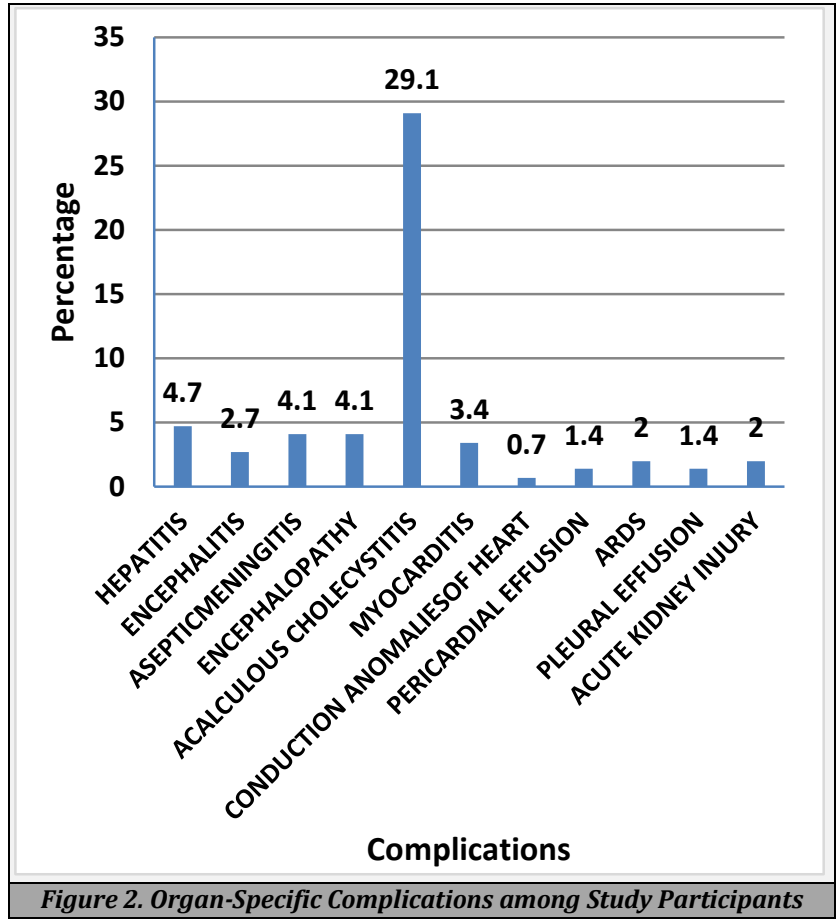

\section{DISCUSSION}

In our cross-sectional study, a total of 148 patients with dengue NS1Ag or dengue IgM positive were included. About 2.5 billion people in both tropical and subtropical region are at a higher risk for developing dengue infection. Approximately $90 \%$ of dengue cases reported worldwide are of children. In this study, most patients were in the age group 21- 30 years. (45.9\%). In previous research that studied the atypical manifestations of dengue outbreak, also the average age of male and female patients was $25.98 \pm 12.96$ years and $26.90 \pm 11.07$ years respectively. ${ }^{23}$ Age is an important risk factor for assessing the risk factors, especially after primary dengue infection. ${ }^{24}$ Dengue, once considered an urban disease, has invaded rural areas due to unplanned urbanization, rapid increase in population, and inefficient waste management system which all favoured the breeding of the vector Aedes aegypti. Unplanned urbanization and densely populated foci are considered to be strong risk factors for the spread of dengue. Dengue has become endemic in rural areas of India as well, increasing the scale of the dengue challenge in the country. A pilot study that conducted in India concluded that areas under urbanization need a stronger surveillance system to monitor dengue outbreak and require implementation of appropriate vector control measures. ${ }^{25}$ Prevention of movement of vectors and viruses because of globalization, development of active surveillance programs, and development of laboratory, entomologic \& epidemiologic facilities are necessary for disease prevention and control. ${ }^{26}$ In our study, the most common symptom was fever followed by other symptoms such as headache, abdominal pain, and vomiting, retro-orbital pain, etc. Altered sensorium, chest pain, breathlessness, cough, and oliguria were also noted in addition to the above said typical symptoms. In this study, $42 \%$ of patients developed atypical manifestations such as acalculous cholecystitis, encephalitis, myocarditis, acute respiratory distress syndrome, acute kidney injury, pericardial effusion, pleural effusion, and conduction anomalies. This is supported by a previous study that investigated the atypical manifestations of dengue outbreaks which reported transverse myelitis as a rare manifestation of DF. In both of these studies, acalculous cholecystitis had the maximum occurrence. ${ }^{23}$ It was diagnosed by sonographic findings and laboratory values. In a previous study it was concluded that acalculous cholecystitis was developed in a significant number of patients and study showed that all the patients were improved with hydration and correction of thrombocytopenia. ${ }^{27}$ Transaminitis ie. SGOT AND SGPT elevation is a common feature in dengue infection. In this study, it was $54.7 \%$ which is comparable to a previous study result.23

The incidence of neurological complications was $10.8 \%$ and aseptic meningitis was the major complication among them. Even though meningitis is a rare presentation of dengue viral fever, a previous study concluded that in endemic areas, dengue should be considered as a probable aetiological agent for the development of meningitis. ${ }^{28}$ Another study conducted in Brazil suggested that simultaneous infection with multiple dengue strains is associated with a higher chance of developing meningitis. ${ }^{29}$ Patients with pleural and pericardial effusion were detected with chest X-ray findings. The plasma leakage into the pleural and pericardial cavities is considered as the aetiology for these symptoms and can be diagnosed with the help of a chest x-ray, ultrasound scan, and serum albumin level $<3.5$ $\mathrm{mg} / \mathrm{dL} \cdot{ }^{30}$ In our study, myocarditis and conduction anomaly developed as a result of dengue infection. The direct action of a virus on cardiomyocytes and the damage induced via the inflammatory mediators released are considered as the mechanism behind these cardiac complications of dengue. ${ }^{31}$ Cardiac arrhythmias are other manifestations of myocardial inflammation. Previous studies reported that dengue is associated with the development of atrial fibrillation, ventricular tachycardia, and atrioventricular block. ${ }^{32,33}$ Histopathological analysis of dengue-associated myocarditis revealed multifocal necrosis, interstitial oedema, and diffuse infiltration of inflammatory cells. ${ }^{34}$ In our study, $2 \%$ of patients had acute kidney injury with raised blood urea and serum creatinine levels. Acute renal failure affects the severity and complications of dengue infection. A previous study showed that acute renal failure is complication of severe dengue infection with a higher side mortality risk. The histopathology examination revealed mesangial proliferation and deposition of immune conjugate deposition in the renal cells. The direct injury by the released inflammatory mediators also could result in acute renal damage. In our study, most of the patients had platelet count in the range of $20000-50000 \mathrm{lakh} / \mathrm{mm} .^{3}$ There is evidence in the works of literature that coagulopathy, vasculopathy, and thrombocytopenia all were associated with severe dengue infection. ${ }^{35}$

This study also found out the association between comorbidities and the presence of organ-specific complications with dengue infection. Patients with DF and co-morbidities seem to be at higher risk of developing complications and/or severe dengue compared to healthier individuals. A previous metanalysis showed that dengue infection in diabetes mellitus (DM) patients could lead to potentially lifethreatening severe complications including organ-specific 
complications. ${ }^{36}$ Cardiac disorders and asthma also were associated with severe complications including end-organ damage. ${ }^{36}$ Acute liver injury is a complication of viral dengue infection, but may not be associated with the degree of viremia. ${ }^{37}$ Hyperlipidaemia is also a proinflammatory state and could lead to severe dengue complications and worsening of dengue infection. Our study also supports the previous study report findings that the co-morbidities could lead to severe dengue complications including end-organ damage.

\section{CONCLUSIONS}

Organ-specific complications in dengue are no longer a rare entity. In this study, many complications were noted such as acalculous cholecystitis (most common), hepatitis, encephalopathy, aseptic meningitis, encephalitis, myocarditis, acute kidney injury, acute respiratory syndrome, pleural effusion, and pericardial effusion. Knowledge of these complications is crucial for early recognition of cases, appropriate treatment and thereby reducing mortality. And also, the presence of co-morbidities in individuals with dengue is a subject underexplored in the scientific literature. Further research in regions with a high prevalence of dengue infection would contribute to a better understanding of the relevance of co-morbidities with severe forms of DF.

\section{Limitations of the Study}

The limitations of this study include lack of co-morbidities and organ-specific complications due to the small sample size and relatively younger age group. As majority of patients were in the young age group, number of patients with comorbidities was less in the sample.

Data sharing statement provided by the authors is available with the full text of this article at jemds.com.

Financial or other competing interests: None.

Disclosure forms provided by the authors are available with the full text of this article at jemds.com.

\section{REFERENCES}

[1] Póvoa TF, Alves AMB, Oliveira CAB, et al. The pathology of severe dengue in multiple organs of human fatal cases: histopathology, ultrastructure and virus replication. PLoS One 2014;9(4):e83386.

[2] WHO. Dengue haemorrhagic fever. Available from: https://scholar.google.com/scholar_lookup?title=Dengu e+hemorrhagic+fever:+diagnosis,+treatment+and+contr ol\&publication_year=1997\&

[3] Barniol J, Gaczkowski R, Barbato EV, et al. Usefulness and applicability of the revised dengue case classification by disease: multi-centre study in 18 countries. BMC Infect Dis 2011;11:106.

[4] Whitehorn J, Farrar J. Dengue. Br Med Bull 2010;95:16173.

[5] Waterman SH, Gubler DJ. Dengue fever. Clin Dermatol 1989;7(1):117-22.
[6] Halstead SB, Lan NT, Myint TT, et al. Dengue hemorrhagic fever in infants: research opportunities ignored. Emerg Infect Dis 2002;8(12):1474-9.

[7] Hasan S, Jamdar SF, Alalowi M, et al. Dengue virus: a global human threat: review of literature. J Int Soc Prev Community Dent 2016;6(1):1-6.

[8] Halstead SB. Observations related to pathogensis of dengue hemorrhagic fever. VI. Hypotheses and discussion. Yale J Biol Med 1970;42(5):350-62.

[9] Guzmán MG, Kourí G. Dengue: an update. Lancet Infect Dis 2002;2(1):33-42.

[10] Khetarpal N, Khanna I. Dengue fever: causes, complications, and vaccine strategies. J Immunol Res 2016;2016:6803098.

[11] Schaefer TJ, Panda PK, Wolford RW. Dengue Fever. In: StatPearls [Internet]. Treasure Island (FL): StatPearls Publishing 2021.

[12] Verma R, Sharma P, Garg RK, et al. Neurological complications of dengue fever: experience from a tertiary center of north India. Ann Indian Acad Neurol 2011;14(4):272-8.

[13] A study on cardiac manifestations of dengue fever. Available from: https://www.japi.org/q2d4b464/astudy-on-cardiac-manifestations-of-dengue-fever

[14] Lee LK, Gan VC, Lee VJ, et al. Clinical relevance and discriminatory value of elevated liver aminotransferase levels for dengue severity. PLoS Negl Trop Dis 2012;6(6):e1676.

[15] de Souza LJ, Alves JG, Nogueira RMR, et al. Aminotransferase changes and acute hepatitis in patients with dengue fever: analysis of 1,585 cases. Braz J Infect Dis 2004;8(2):156-63.

[16] Saha AK, Maitra S, Hazra SC. Spectrum of hepatic dysfunction in 2012 dengue epidemic in Kolkata, West Bengal. Indian J Gastroenterol 2013;32(6):400-3.

[17] Garg RK, Malhotra HS, Jain A, et al. Dengue-associated neuromuscular complications. Neurol India 2015;63(4):497-516.

[18] Lizarraga KJ, Nayer A. Dengue-associated kidney disease. J Nephropathol 2014;3(2):57-62.

[19] Mohamed NA, El-Raoof EA, Ibraheem HA. Respiratory manifestations of dengue fever in Taiz-Yemen. Egyptian Journal of Chest Diseases and Tuberculosis 2013;62(2):319-23.

[20] Yip VCH, Sanjay S, Koh YT. Ophthalmic complications of dengue fever: a systematic review. Ophthalmol Ther 2012;1(1):2.

[21] Badawi A, Velummailum R, Ryoo SG, et al. Prevalence of chronic comorbidities in dengue fever and West Nile virus: a systematic review and meta-analysis. PLoS One 2018;13(7):e0200200.

[22] Dhivya P, Nagesh GN, Jayaramachandran S. Effect of co morbidities on the clinical outcome of dengue fever. International Journal of Advances in Medicine 2019;6(3):750-5.

[23] Ahlawat RS, Kalra T. Atypical manifestations of dengue fever in a recent dengue outbreak. Annals of Tropical Medicine and Public Health 2017;10(6):1448-51.

[24] Egger JR, Coleman PG. Age and clinical dengue illness. Emerg Infect Dis 2007;13(6):924-7. 
[25] Shah PS, Deoshatwar A, Karad S, et al. Seroprevalence of dengue in a rural and an urbanized village: a pilot study from rural western India. J Vector Borne Dis 2017;54(2):172-6.

[26] Gubler DJ. Dengue, urbanization and globalization: the unholy trinity of the 21st century. Trop Med Health 2011;39(4 Suppl):3-11.

[27] Bhatty S, Shaikh NA, Fatima M, et al. Acute acalculous cholecystitis in dengue fever. J Pak Med Assoc 2009;59(8):519-21.

[28] Goswami RP, Mukherjee A, Biswas T, et al. Two cases of dengue meningitis: a rare first presentation. J Infect Dev Ctries 2012;6(2):208-11.

[29] Marinho PES, de Oliveira DB, Candiani TMS, et al. Meningitis associated with simultaneous infection by multiple dengue virus serotypes in children, Brazil. Emerg Infect Dis 2017;23(1):115-8.

[30] Kalayanarooj S. Clinical manifestations and management of dengue/DHF/DSS. Trop Med Health 2011;3944 Suppl):83-7.

[31] Salgado DM, Eltit JM, Mansfield K et al. Heart and skeletal muscle are targets of dengue virus infection. Pediatr Infect Dis J 2010;29(3):238-42.
[32] Veloso HH, Ferreira Júnior JA, de Paiva JMB, et al. Acute atrial fibrillation during dengue hemorrhagic fever. Braz J Infect Dis 2003;7(6):418-22.

[33] Donegani E, Briceño J. Disorders of atrio-ventricular conduction in patients with hemorrhagic dengue. Minerva Cardioangiol 1986;34(7-8):477-80.

[34] Miranda $\mathrm{CH}$, de Borges MC, Schmidt A, et al. A case presentation of a fatal dengue myocarditis showing evidence for dengue virus-induced lesion. Eur Heart J Acute Cardiovasc Care 2013;2(2):127-30.

[35] de Azeredo EL, Monteiro RQ, de Oliveira Pinto LM. Thrombocytopenia in dengue: interrelationship between virus and the imbalance between coagulation and fibrinolysis and inflammatory mediators. Mediators Inflamm 2015;2015:313842.

[36] Pang J, Hsu JP, Yeo TW, et al. Diabetes, cardiac disorders and asthma as risk factors for severe organ involvement among adult dengue patients: a matched case-control study. Sci Rep 2017;7:39872.

[37] Fernando S, Wijewickrama A, Gomes L, et al. Patterns and causes of liver involvement in acute dengue infection. BMC Infectious Diseases 2016;16(1):319. 